\title{
3T MRI Reveals Extra- and Intracranial Involvement in Giant Cell Arteritis
}

\author{
S. Siemonsen, C. Brekenfeld, B. Holst, A.-K. Kaufmann-Buehler, J. Fiehler, and T.A. Bley
}

\begin{abstract}
BACKGROUND AND PURPOSE: The frequency and amount of intracranial, intradural inflammatory vessel wall enhancement in giant cell arteritis remain unclear. The purpose of this work was to prospectively assess the intracranial extent of vasculitic changes in patients with giant cell arteritis using a dedicated MR imaging protocol optimized for assessment of mural changes of intracranial arteries.
\end{abstract}

MATERIALS AND METHODS: Twenty-eight patients with suspected giant cell arteritis underwent 3T MR imaging. Imaging included a fat-saturated TIWI pre- and postcontrast application optimized for assessment of intradural vessel wall enhancement and high-resolution fat-saturated TIWI to evaluate superficial extracranial vessels. Temporal artery biopsies were available in 11 cases. Vessel wall enhancement of intradural and extracranial vessels was evaluated by 2 observers independently.

RESULTS: Twenty patients had giant cell arteritis; 9 cases were biopsy-proved. Clear vessel wall enhancement of superficial extracranial and intradural internal carotid arteries was detected in 16 and 10 patients, respectively. Slight vessel wall enhancement of the vertebral arteries was seen. Of 9 patients with giant cell arteritis with vessel occlusion or stenosis, 2 presented with cerebral ischemic infarcts. Vessel occlusion or stenosis site coincided with the location of vessel wall enhancement of the vertebral arteries in 4 patients and of the intradural ICA in 1 patient.

CONCLUSIONS: Vessel wall enhancement of intradural arteries, mainly the ICA, can be regularly found in patients with giant cell arteritis. Mural inflammatory changes of the intradural ICA detected on MR imaging may identify a subgroup of patients with giant cell arteritis and should be further evaluated in clinical studies.

ABBREVIATIONS: fsTIWI = fat-saturated TIWI; GCA = giant cell arteritis; $T A B=$ temporal artery biopsy; $V W E=$ vessel wall enhancement

G iant cell arteritis (GCA) commonly affects large- and medium-sized arteries with predominance in the aorta and the supra-aortic vessels such as the subclavian, carotid, and axillary arteries and the superficial cranial arteries, among others. ${ }^{1,2}$ Recent imaging studies have shown inflammatory involvement of the aorta in up to $65 \%$ and of the subclavian arteries in up to $35 \%$ of patients. ${ }^{3,4}$

The extent of vasculitic changes to the intracranial vasculature

Received May 12, 2014; accepted after revision July 13.

From the Department of Diagnostic and Interventional Neuroradiology (S.S., T.A.B.), University Medical Center Hamburg-Eppendorf, Hamburg, Germany; and Department of Diagnostic and Interventional Radiology (C.B., B.H., A.-K.K.-B., J.F.) University Hospital of Würzburg, Würzburg, Germany.

This work was supported by Deutsche Forschungsgemeinschaft, DFG: BL 1132/1-1.

Please address correspondence to Susanne Siemonsen, MD, Department of Diagnostic and Interventional Neuroradiology, University Medical Center HamburgEppendorf, Martinistr 52, 20246 Hamburg, Germany; e-mail: s.siemonsen@ uke.uni-hamburg.de

- Indicates open access to non-subscribers at www.ajnr.org

http://dx.doi.org/10.3174/ajnr.A4086 is discussed controversially and is currently not fully understood. Not a single intracranial artery was involved in a postmortem study of patients who died during the phase of active GCA when the extracranial superficial temporal and ophthalmic arteries were severely inflamed. The different composition of the intracranial arteries with less elastic tissue in the media compared with the extracranial arteries may correlate with the inflammatory activity in GCA. ${ }^{5}$ However, a more recent article indicated that intracranial involvement in GCA does occur, presumably less frequently. ${ }^{6}$

High-resolution MR imaging studies have been proved to reliably detect mural inflammation such as mural thickening and increased mural contrast enhancement of the superficial temporal and occipital arteries in biopsy-proved GCA. ${ }^{7,8}$ Diagnostic criteria for detecting intracranial vasculitis are intramural contrast uptake, in most cases combined with wall thickening. ${ }^{9}$ These criteria are valid for patients presenting with primary angiitis of the central nervous system, childhood primary angiitis of the central nervous system or GCA, and other causes of central nervous system vasculitis. $^{10}$ 


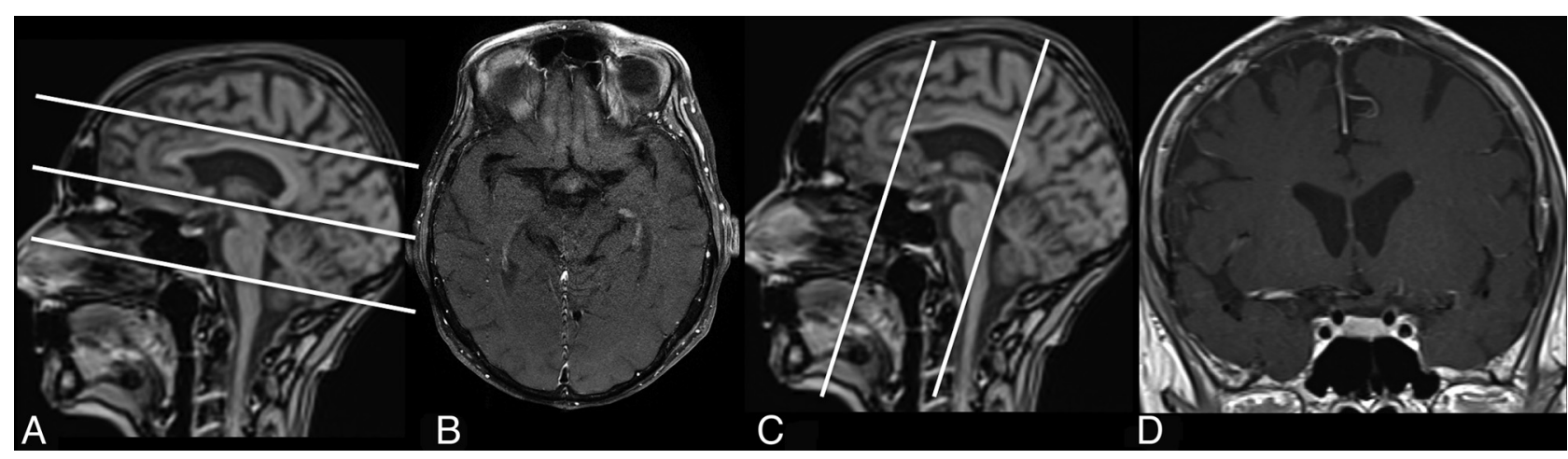

FIG 1. Fat-saturated TIWI scans post-contrast medium application and corresponding scouts optimized for assessment of intradural VWE (C and $D$ ), and high-resolution fsTIWI scans to evaluate superficial extracranial vessels ( $A$ and $B$ ).

A retrospective analysis of high-resolution $\mathrm{MR}$ imaging of the superficial cranial arteries of 50 patients with GCA did not reveal any inflammatory mural changes of the intracranial arteries of the circle of Willis. ${ }^{11}$ However, the MR imaging protocol in that particular study was optimized to depict mural inflammation of the superficial cranial arteries and was not specifically adjusted for visualization of the intradural arteries. The purpose of this work was to prospectively assess the intracranial extent of vasculitic changes in patients with GCA using a dedicated MR imaging protocol optimized for assessment of mural changes of intracranial arteries.

\section{MATERIALS AND METHODS \\ Patients}

Twenty-eight consecutive patients (21 women, 7 men; 62-91 years of age; mean, 74 years) with clinically suspected GCA were referred to our institution between 2012 and 2013 and were prospectively included in our study. The study was approved by the local ethics review committee and is in accordance with the Declaration of Helsinki. Written informed consent was obtained from all subjects. Patients were referred for MR imaging by rheumatologists, ophthalmologists, and neurologists experienced in diagnosing and treating patients with GCA. All patients underwent an MR imaging examination of the brain, including dedicated sequences optimized for assessment of mural changes of the extracranial and intracranial arterial vessel walls.

Temporal artery biopsy (TAB) was performed in 11 of the 28 patients. Biopsy specimens were obtained from the frontal branch of the superficial temporal artery that was clinically most prominently affected. MR imaging was performed before TAB in all except 1 patient. Twenty patients were found to be positive for GCA (GCA-positive) according to the clinician's final diagnosis. In 11 of these patients positive for GCA, diagnosis was validated only by fulfillment of the American College of Rheumatology clinical criteria for GCA, ${ }^{12}$ and in 9 , by positive histopathologic findings of multinucleated giant cells or mononuclear cells infiltrating the wall of the frontal branch of the superficial temporal artery. In 1 patient positive for GCA, histology yielded false-negative results.

Five patients were found negative for GCA (GCA-negative) according to the clinician's final diagnosis. In 3 patients, the definite diagnosis remained unclear; these patients were excluded from further analysis.
Of the 20 patients positive for GCA, 18 began corticosteroid treatment $1-8$ days (median, 3.5 days) before MR imaging, while 2 had not received any corticosteroid treatment.

C-reactive protein level and erythrocyte sedimentation rate were available at admission in all 20 and 19 patients positive for GCA, respectively. C-reactive protein level was available in all patients negative for GCA; erythrocyte sedimentation rate, in 4 of 5 patients. In 1 patient, the exact erythrocyte sedimentation rate was not documented, but a clear elevation was noted.

\section{MR Imaging Examination and Protocol}

All MR imaging was performed on a 3T system (Magnetom Skyra; Siemens, Erlangen, Germany) with a 20-channel head coil. The MR imaging protocol included diffusion-weighted imaging; TOF angiography; fluid-attenuated inversion recovery; fat-saturated T1WI (fsT1WI) scans before and after gadolinium administration, optimized for the assessment of intradural vessel wall enhancement (VWE); and high-resolution fsT1WI scans to evaluate mural changes of superficial extracranial vessels. In 16 patients, contrast-enhanced MR angiography of the cervical arteries was performed. All patients received $16 \mathrm{~mL}$ of a gadolinium-based contrast agent (gadopentetate dimeglumine, $0.5 \mathrm{mmol} / \mathrm{mL}$, Magnograf; Marotrast, Jena, Germany).

For DWI, a single-shot, spin-echo, echo-planar imaging sequence was used, and images were collected with $b=0$ and 1000 $\mathrm{s} / \mathrm{mm}^{2}$. TOF angiography was performed by using $3 \mathrm{D}$ fast imaging with a steady-state precession sequence with venous saturation, a magnetization transfer saturation pulse, and a tilted optimized nonsaturation excitation-up pulse. Coronal oriented multisection fsT1WI spin-echo sequences were acquired before and 3 minutes after venous injection of a gadolinium-based contrast agent. The image localizer was positioned perpendicular to the orientation of the vessel. Postcontrast, multisection fsT1WI spin-echo images were acquired with an in-plane resolution of $0.2 \times 0.2 \mathrm{~mm}$. Two consecutive acquisitions were performed covering $10.8 \mathrm{~cm}$ in a craniocaudal direction with the lowest section leveling with the earlobes (Fig 1). Details on sequence parameters are shown in Table 1.

\section{MR Imaging Evaluation}

The extracranial vessels (ie, the frontal and parietal branches of the superficial temporal and the occipital arteries) were evaluated by 2 experienced neuro-/radiologists (S.S. and T.A.B.) on fsT1WI 
Table 1: MRI sequence parameters

\begin{tabular}{|c|c|c|c|c|c|c|c|c|c|c|}
\hline Sequence & TE (ms) & TR (ms) & $\mathrm{TI}$ (ms) & FOV (mm) & Matrix & Sections & $\begin{array}{c}\text { Section } \\
\text { Thickness (mm) }\end{array}$ & Flip Angle & Orientation & Gap \\
\hline FLAIR & 90 & 9000 & 2500 & 230 & $270 \times 320$ & 25 & 5 & & Axial & $10 \%$ \\
\hline DWI & 90 & 7900 & & 230 & $256 \times 256$ & 25 & 5 & & Axial & $10 \%$ \\
\hline TOF & 3.43 & 21 & & 200 & $384 \times 331$ & 136 & 5 & $18^{\circ}$ & Axial & \\
\hline fsTIWI pre- and post-GD & 11 & 549 & & 200 & $320 \times 320$ & 20 & 2 & $150^{\circ}$ & Coronal & \\
\hline $2 \times$ fsTIWI post-GD & 22 & 500 & & 200 & $1024 \times 768$ & 18 & 3 & & Axial & \\
\hline
\end{tabular}

Note:-GD indicates gadolinium injection.
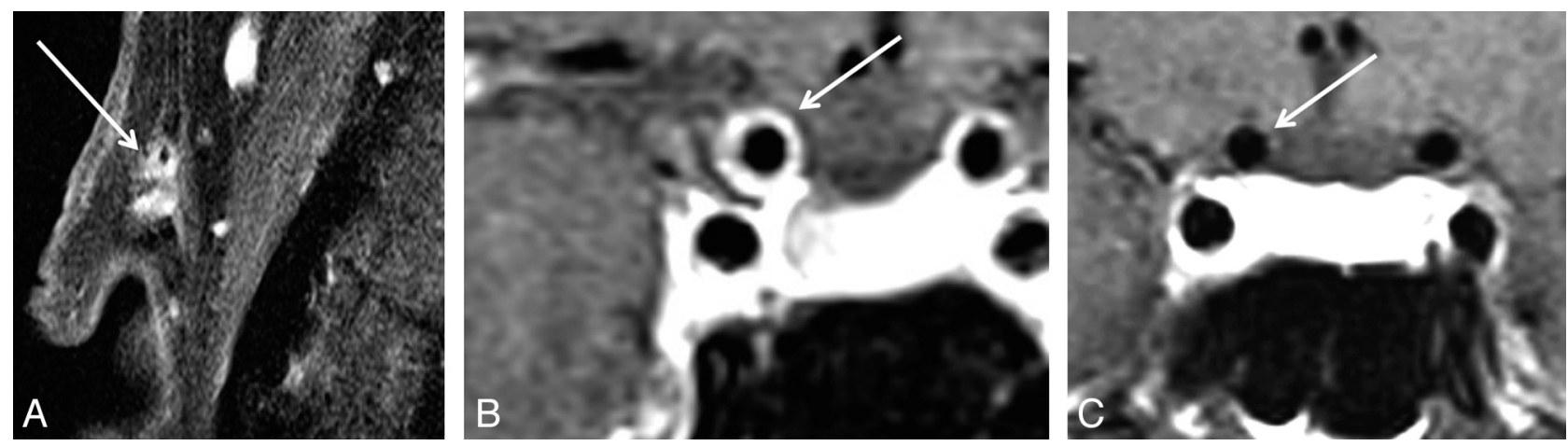

FIG 2. A patient with GCA with vessel wall enhancement of extracranial $(A)$ and intracranial intradural internal carotid arteries (B). In contrast, patient with GCA without VWE of intracranial intradural arteries (C).

high-resolution MR images. Both readers were blinded to the clinical, biopsy, and laboratory findings. As previously proposed, mural thickening and prominent contrast enhancement of the vessel wall were considered signs of mural inflammation. ${ }^{7} \mathrm{MRI}$ signs of extracranial vessel wall inflammation and thickening were documented by each rater separately for all 3 locations bilaterally. In case of differing results, a consensus rating was established. Depending on the consensus, we dichotomized patients in the following manner: Patients with signs of inflammation of any of the evaluated branches of the superficial temporal artery or occipital artery were grouped into MR-GCA-positive, and patients with negative ratings, into MR-GCA-negative.

Intradural vessels of patients were evaluated on fsT1WI before and after contrast agent administration independently for signs of vessel wall enhancement and/or thickening indicating mural inflammation by 2 experienced neuroradiologists (S.S. and C.B.) who were blinded to the patient's clinical information and diagnosis during the rating. The wall of the internal carotid artery was rated as positive contrast-enhancing (MR-ICA-positive) in cases where a circular enhancement was clearly visible distant from the cavernous sinus (ie, at least 2 sections distant from the last section including a part of the cavernous ICA; Fig 2). Otherwise patients were rated as MR-ICA-negative. The vertebral arteries were evaluated at least $0.5 \mathrm{~cm}$ distant from entering the spinal canal, close to the confluens to avoid misjudging the accompanying venous vessels at the site of dural penetration. Similarly, the anterior cerebral artery, posterior cerebral artery, and middle cerebral artery were evaluated for VWE. Any sign of clear enhancement in comparison with corresponding native scans was rated as positive. In case of differing results, a consensus rating was established by a third experienced neuroradiologist (B.H.).

The V4 segment of the vertebral artery was not assessable in 6 patients due to positioning of the localizer but was included in the analysis in all other cases. Similarly, the basilar artery was prone to pulsation artifacts and was not assessable in 3 patients. In addition, cerebral MR images and angiographies were evaluated by 1 experienced neuroradiologist (S.S.) for acute ischemic infarction visible on DWI, signs of previous infarction, or other pathologies on FLAIR images. TOF angiography of intracranial vessels and contrast-enhanced angiography of cervical vessels if available were evaluated for stenosis or vessel occlusion. These ratings were performed independent from intracranial and extracranial ratings, and evaluators were blinded to the results of ratings of extraor intracranial VWE.

\section{Statistical Analysis}

The interobserver agreement for extracranial and intracranial vessel involvement was evaluated with the Cohen $\kappa$ test and magnitude guidelines applied according to Landis and Koch. ${ }^{13}$ The median values and SD for age, C-reactive protein level, and erythrocyte sedimentation rate were calculated for the patient groups (GCA-positive and GCA-negative). The diagnosis of GCA by MR imaging was compared with the clinical diagnosis. In addition, association of extracranial and intracranial mural inflammation with the GCA diagnosis was evaluated by using the $\chi^{2}$ test. The sensitivity, specificity, and positive and negative predictive values were calculated and reported with corresponding confidence intervals and $P$ values (Fisher exact test). Statistical analysis was conducted by using $\mathrm{R}$ statistical computing software, Version 3.0.0 (http://www.r-project.org/).

\section{RESULTS}

\section{Clinical Findings of Patients}

The mean \pm SD level of C-reactive protein at admission was $80.5 \pm 71.6 \mathrm{mg} / \mathrm{dL}$ in patients positive for GCA and $36.6 \pm 65.7$ $\mathrm{mg} / \mathrm{dL}$ in patients negative for GCA. The mean \pm SD erythrocyte sedimentation rate at admission, if available, was $70.58 \pm 31.57$ $\mathrm{mm} / \mathrm{h}$ in patients positive for GCA and $45.50 \pm 38.79 \mathrm{~mm} / \mathrm{h}$ in 
Table 2: Patient details on clinical and MRI findings

\begin{tabular}{|c|c|c|c|c|c|c|c|c|}
\hline Patient No. & Age (yr) & Sex & GCA-Diagnosis & MR-GCA & MR-ICA & TAB & $\begin{array}{l}\text { Initial Presentation } \\
\text { to MRI (days) }\end{array}$ & $\begin{array}{l}\text { ROIDS to MRI } \\
\text { (days) }\end{array}$ \\
\hline 1 & 77 & $\mathrm{~F}$ & Positive & Positive & $R+L$ & Positive & 3 & 2 \\
\hline 2 & 72 & $M$ & Positive & Positive & $\mathrm{R}+\mathrm{L}$ & Positive & 2 & 1 \\
\hline 3 & 81 & $\mathrm{~F}$ & Positive & Negative & Negative & NA & 2 & 2 \\
\hline 4 & 74 & $M$ & Positive & Positive & $\mathrm{L}$ & NA & 5 & 4 \\
\hline 5 & 71 & $\mathrm{~F}$ & Positive & Positive & $\mathrm{R}+\mathrm{L}$ & Positive & 8 & 8 \\
\hline 6 & 70 & $\mathrm{~F}$ & Positive & Positive & $\mathrm{L}$ & NA & 4 & 4 \\
\hline 7 & 66 & $\mathrm{~F}$ & Positive & Positive & Negative & Positive & 1 & 1 \\
\hline 8 & 75 & $\mathrm{~F}$ & Positive & Positive & $\mathrm{L}$ & NA & 2 & 2 \\
\hline 9 & 71 & $M$ & Positive & Negative & Negative & NA & 6 & 5 \\
\hline 10 & 73 & $\mathrm{~F}$ & Positive & Negative & Negative & Positive & 1 & 1 \\
\hline 11 & 91 & M & Positive & Positive & Negative & NA & 6 & 6 \\
\hline 12 & 72 & $\mathrm{~F}$ & Negative & Negative & Negative & NA & 4 & 0 \\
\hline 13 & 73 & $M$ & Positive & Positive & $\mathrm{R}+\mathrm{L}$ & Positive & 2 & 0 \\
\hline 14 & 78 & $\mathrm{~F}$ & Negative & Negative & Negative & Negative & 1 & 1 \\
\hline 15 & 63 & $\mathrm{~F}$ & Positive & Positive & Negative & NA & 2 & 0 \\
\hline 16 & 86 & $\mathrm{~F}$ & Positive & Positive & Negative & Positive & 4 & 4 \\
\hline 17 & 64 & $M$ & Positive & Positive & Negative & NA & 4 & 3 \\
\hline 18 & 68 & $M$ & Positive & Positive & L & Positive & 3 & 3 \\
\hline 19 & 75 & $\mathrm{~F}$ & Positive & Positive & $R+L$ & NA & 2 & 1 \\
\hline 20 & 62 & $\mathrm{~F}$ & Positive & Positive & $\mathrm{L}$ & NA & 7 & 7 \\
\hline 21 & 70 & $\mathrm{~F}$ & Negative & Negative & Negative & NA & 4 & NA \\
\hline 22 & 83 & $\mathrm{~F}$ & Negative & Negative & Negative & NA & 9 & NA \\
\hline 23 & 87 & $\mathrm{~F}$ & Positive & Negative & Negative & Negative & 8 & 8 \\
\hline 24 & 78 & $\mathrm{~F}$ & Positive & Positive & Negative & Positive & 4 & 4 \\
\hline 25 & 79 & $\mathrm{~F}$ & Negative & Positive & Negative & NA & 6 & 1 \\
\hline
\end{tabular}

Note:-R indicates right; L, left; ROIDS, corticosteroids; NA, not applicable.

patients negative for GCA. There was no significant difference between the groups $(P>.05)$. On admission, 3 of the patients positive for GCA presented with temporal artery tenderness to palpation; 4, with visual impairment and new headache; 7, with temporal artery tenderness to palpation in combination with visual impairment; 3 , with recent weight loss and new headache; and 3, with new headache and weight loss in combination with dizziness and gait abnormalities.

\section{Evaluation of Extracranial Arteries}

The MR images had good diagnostic quality in 25 cases; only 1 patient terminated the scan before the second slab of the fsT1WI for evaluation of extracranial vessels was finished; therefore, in this patient, only the lower slab was available for evaluation. In 1 case, assessment of the extracranial arteries was slightly impaired due to motion artifacts. Nevertheless, the superficial occipital artery, the frontal branch of the superficial temporal artery, and the parietal branch of the superficial temporal artery could be clearly depicted in all scans.

In 1 patient (patient 23) with false-negative biopsy results, the biopsy specimen was extracted from the left temporal artery 5 days before MR imaging, and this sample was found to be negative for GCA. The diagnosis of GCA in this patient was validated by the American College of Rheumatology clinical criteria. MR imaging also revealed no signs of mural inflammation, only slight diffuse contrast enhancement adjacent to the left temporal artery where the sample was harvested, consistent with granulation tissue. Nevertheless, in this patient, treatment with corticosteroids was initiated 3 days before biopsy and 8 days before MR imaging.

The Cohen $\kappa$ for interrater reliability for assessment of MRGCA-positive and MR-GCA-negative was almost perfect with
$0.905(P<.001)$. Nevertheless, $\kappa$ values for ratings of 1 -sided or bilateral involvement of the frontal branches of the superficial temporal artery were substantial at $0.662(P<.01)$; for the parietal branches, moderate at $0.51(P<.01)$; and for involvement of the occipital artery, moderate at $0.603(P<.01)$.

Among the 150 extracranial arteries examined, 85 (56.7\%) were considered inflamed and $65(43.3 \%)$, to be unaffected.

Of the 20 patients positive for GCA, 16 were rated as MRGCA-positive, while 4 were rated MR-GCA-negative and were therefore false-negatives. Three of these patients negative for MRGCA did not show any clear VWE in any of the evaluated arteries. One of the patients positive for GCA showed solitary VWE of only the frontal branch of the left superficial temporal artery. In all other patients MR-GCA-positive, at least 2 extracranial arteries were involved. Bilateral involvement was found in all true-positive cases. One patient negative for GCA was rated as MR-GCApositive and therefore false-positive. The frontal branches of the superficial temporal artery were involved in 16 of 20 patients positive for GCA; the parietal branches, in 11; and the occipital artery, in 13, respectively. Details for each patient are presented in Table 2.

Sensitivity and specificity of MR-GCA ratings for the detection of GCA diagnosis were 0.80 (95\% CI, 0.56-0.94) and 0.80 (95\% CI, $0.28-0.99)$, respectively. The positive predictive value was 0.94 (95\% CI, 0.71-0.99), and the negative predictive value was 0.50 (95\% CI, 0.15-0.84) with $P<.05$.

Patients with false-negative findings (patients 3, 9, 10, and 23) were treated with corticosteroids between 1 and 8 days (mean, 4 days) before MR imaging.

In the subgroup of the 9 patients with biopsy-proved GCA, 8 were MR-GCA-positive and 1 was rated MR-GCA-negative. In 
the subgroup of patients with a clinical diagnosis of GCA, 8 patients were rated as MR-GCA-positive, while 3 were rated as MR-GCA-negative.

\section{Evaluation of Intradural Arteries}

The Cohen $\kappa$ for evaluation of intradural ICA VWE was almost perfect with 0.839 . Bilateral enhancement was detected in 5 of 25 patients, and unilateral enhancement, in 5 patients, whereas in 15 patients, there was no enhancement (MR-ICA-negative). Therefore 10 patients displayed signs of uni- or bilateral VWE of the intradural ICA (MR-ICA-positive). In 9 of these patients, VWE was accompanied by vessel wall thickening. Of the 10 patients positive for MR-ICA, all were positive for GCA. Of the 15 patients negative for MR-ICA, 5 were negative for GCA and 10 were positive for GCA. There was no significant difference in C-reactive protein and erythrocyte sedimentation rate between the 2 groups $(P>.05)$.

Sensitivity and specificity of MR-ICA ratings for the prediction of GCA diagnosis were 0.50 (95\% CI, 0.25-0.70) and 0.99 (95\% CI, 0.40-0.99), respectively. The positive predictive value was 0.99 (95\% CI, 0.63-0.99), and the negative predictive value was 0.34 (95\% CI, 0.11-0.59) with $P>.05$.

There was a significant association of MR-GCA-positive and MR-ICA-positive findings $(P<.05)$ when including all 25 pa-

Table 3: Ratings of extra- and intracranial vessel wall inflammation and grouping according to GCA diagnosis

\begin{tabular}{lccc}
\hline & MR-ICA-Positive & MR-ICA-Negative & Total \\
\hline GCA-Positive & & 6 & 20 \\
MR-GCA-Positive & 10 & 4 & 16 \\
MR-GCA-Negative & 0 & & 4 \\
GCA-Negative & & 0 & 5 \\
MR-GCA-Positive & 1 & 4 & 1 \\
MR-GCA-Negative & 0 & & 4 \\
\hline
\end{tabular}

tients. Within the patients positive for GCA, there was no significant association between MR-GCA-positive and MR-ICA-positive findings (Table 3).

In the subgroup of the 9 patients with biopsy-proved GCA, 5 were MR-ICA-positive and 4 were MR-GCA-negative. In the subgroup of patients with a clinical diagnosis of GCA, 5 patients were rated as MR-ICA-positive, while 6 were rated as MR-GCA-negative.

In addition, 5 patients presented with bilateral VWE of the vertebral arteries (all GCA-positive); 4, with VWE of the left (3 of 4 GCA-positive) vertebral arteries, adding to 8 venous vessels at the site of dural penetration ratings (7 positive for GCA, 1 negative for GCA). Therefore, 11 cases were rated as MR-vertebral artery-negative ( 3 negative for GCA, 8 positive for GCA). VWE of the basilar artery was detected in none of our patients. One GCApositive patient displayed VWE of the left M1 segment (MR-MCA-positive).

\section{Vessel Stenosis or Occlusion}

In total, 13 patients did not present with any site of vessel occlusion or stenosis (2 GCA-negative and 11 GCA-positive). Of the remaining 12 patients, 9 were positive for GCA and 3, negative for GCA. Of these, 6 patients showed V4 stenosis (3 right, 1 left, 2 bilateral), while 2 patients presented with V4 occlusion. ICA siphon stenosis was observed in 4 ( 1 right, 1 left, 2 bilateral); right ICA occlusion, in 1 patient. Vessel occlusion or stenosis site coincided with the VWE location of the vertebral arteries in 4 (Fig 3) and of the intradural ICA in 1 patient, with right intracavernous ICA occlusion sparing the distal carotid-T.

\section{Brain Parenchymal Findings}

Of the 25 included patients, 2 presented with pathologic brain parenchymal findings on MR imaging. Both patients were GCA-

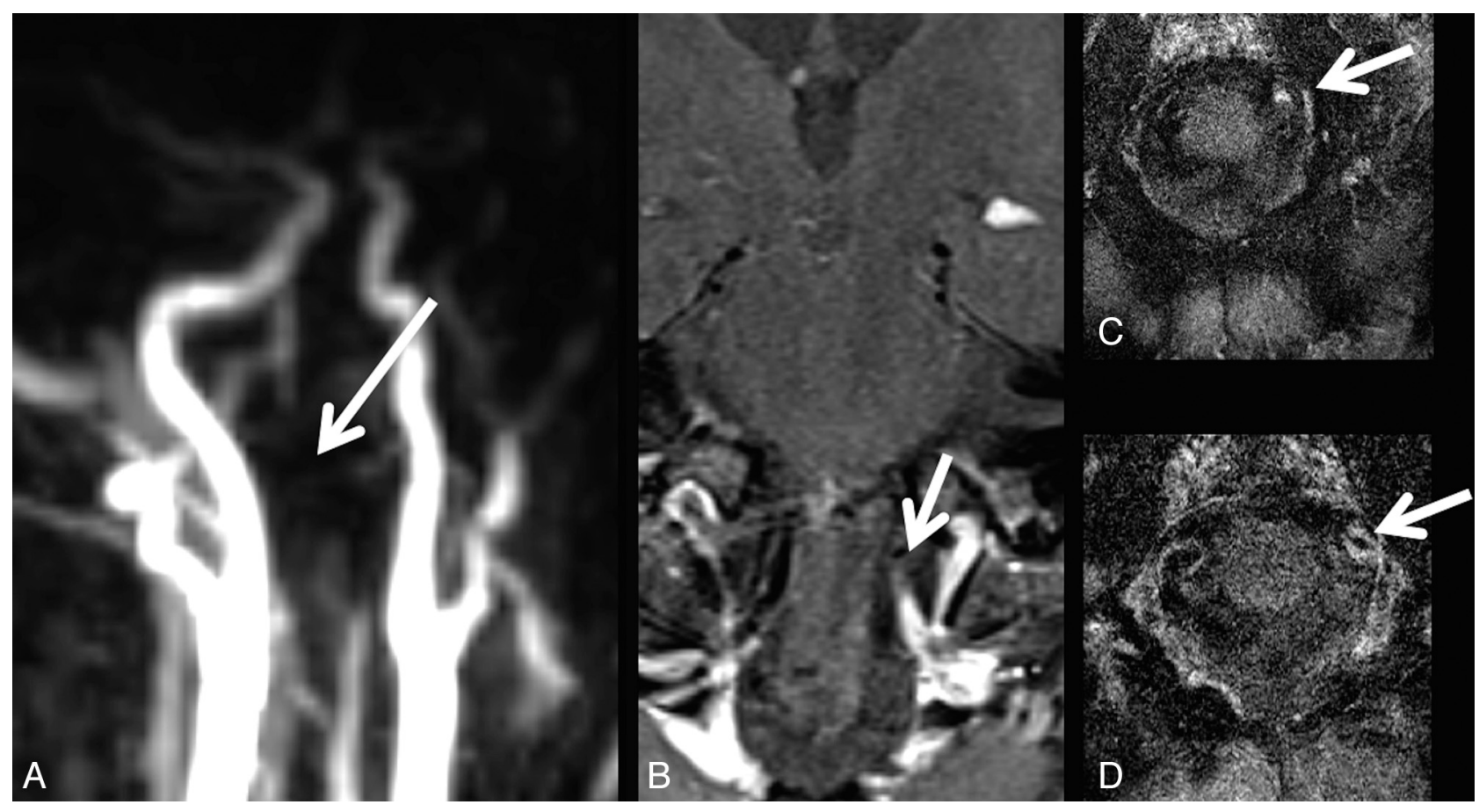

FIG 3. A patient with GCA with occlusion of the left vertebral artery $(A)$ and corresponding vessel wall enhancement $(B-D)$. 
positive. One patient (patient 5) presented with subacute ischemic infarcts of the cerebellum and the right head of the caudate nucleus and cortical watershed infarction caused by right ICA occlusion. Another patient presented with a history of cerebellar ischemic infarction combined with right V4 segment occlusion and left V3/V4 stenosis. Additionally, 11 patients had imaging signs indicative of degenerative cerebral microangiopathy.

\section{DISCUSSION}

In our study, most patients positive for GCA were characterized through clear signs of mural inflammation of at least 2 affected extracranial arteries. These observations are in line with previous studies reporting mural inflammation of the temporal artery in patients with GCA depicted by high-resolution MR imaging. ${ }^{8,14-16}$ Even patients who received corticosteroids for $>2$ days before MR imaging displayed signs of mural inflammation. A sensitivity of 0.80 and a specificity of 0.80 for the detection of GCA by using this imaging protocol were comparable with recent studies with reported values of sensitivities between $0.80 \%$ and $0.85 \%$ and specificities ranging from $0.71 \%$ to $0.95 \% .^{7,17}$ This result might be accounted for by the differing number of days that patients received corticosteroids before MR imaging examination.

Irrespective of extracranial VWE, we observed clear VWE of the intradural ICA in at least $50 \%$ of patients positive for GCA, while none of the patients negative for GCA presented with this finding. Additionally, signs of mural inflammation of the vertebral arteries were observed in 4 patients positive for GCA and of the proximal MCA in 1 patient. Involvement of intracranial and intradural arteries has so far been reported by only a small number of case reports. ${ }^{18-20}$ A review of several published GCA cases with intracranial and intradural involvement concluded that these might only represent a subset of patients with GCA and they seem to occur rarely. Likewise, cerebral infarcts have been reported in only $3 \%-4 \%$ of patients with GCA. ${ }^{21,22}$ In comparison, we observed pathologic brain parenchymal findings with a higher incidence $(15 \%)$, a rate similar to that in another study reporting an incidence of $14.3 \%$. Nevertheless ischemic infarcts still seem to be a rare finding in GCA. Several studies suggest that ischemic infarctions in cases of GCA are caused by stenosis or occlusion mainly of extradural vertebral and carotid arteries rather than intracranial or intradural vasculitis. ${ }^{5,23-28}$

Accordingly, in our study, VWE of the brain-supplying arteries coincided more frequently with corresponding arterial occlusion or stenosis in cases in which the vertebral arteries were affected rather than in cases of involvement of the intradural ICA. VWE of the intradural ICA was observed in a number of patients positive for GCA, but only 1 case corresponding to local vessel occlusion was detected. Correspondingly, cerebrovascular ischemic events in our patient group were only observed in patients with concomitant pathology of the extradural or extracranial vessels. Therefore, unlike in other forms of primary or secondary cerebral vasculitis, ${ }^{10}$ in patients positive for GCA, VWE of the intracranial ICA was not regularly associated with corresponding stenosis or occlusion.

So far, larger studies focusing on GCA have not reported involvement of any intradural arteries. ${ }^{7,8,10,11,14}$ This discrepancy might be caused by the differing study designs and MR imaging protocols that are typically based on the focus of the study. In general, deferring sequence parameters optimized for visualization of mural inflammation of intra- and extracranial vessels are mandatory. While the MR imaging sequence used for the evaluation of extracranial vessels is technically focused on high-resolution and contrast of the skull and scalp region, T1WI for the evaluation of intracranial vessels is focused on reduction of pulsation artifacts, a high signal-to-noise ratio, and contrast within the FOV center (Fig 1).9,10

In patients positive for GCA, involvement of intradural vessels coincided rarely with stenosis or occlusion and the occurrence of associated ischemic infarctions. Therefore, former studies might not have recognized mural inflammation of the intradural arteries in patients with GCA because in most studies, patients were only included and examined extensively provided they presented with ischemic stroke. ${ }^{18,19,23,29}$ Nevertheless, most of these patients did not undergo an MR imaging examination including sequences adapted for evaluation of intracranial VWE. 6,11

Moreover, postmortem examination can only be performed in patients with GCA with lethal complications (eg, cerebrovascular ischemic events) but not systematically. In addition, postmortem analysis in patients with GCA cannot guarantee an acute state of inflammation of the analyzed tissue. Still, at least intradural involvement of the vertebral arteries was reported in single postmortem cases. ${ }^{5,6}$ Another postmortem study reported 1 of 4 patients with GCA with generalized vasculitis in large and small cerebral vessels. ${ }^{19}$ Accordingly, studies focused on primary or secondary vasculitis with cerebral involvement generally do not include patients with a GCA diagnosis. Nevertheless, at least 1 study reported a case of biopsy-proved GCA with VWE of the right carotid-T with co-localized vessel stenosis or occlusion and acute ischemic infarction. ${ }^{10}$ Another group reported a case of a GCAinduced aneurysm of the MCA. ${ }^{30}$

However, none of these studies systematically evaluated prospectively recruited patients with GCA. In comparison with the high sensitivity and specificity of mural inflammation of extracranial vessels for a GCA diagnosis, the sensitivity for ICA involvement was very low, while specificity was high. Therefore, signs of inflammation of the intradural arteries are not potentially a diagnostic criterion. Nevertheless, this imaging sign seems to be highly specific and might therefore identify a subgroup of patients with GCA.

A limitation of our study was that most of our patients had already received corticosteroids for numerous days before MR imaging. Initially, there might have been more cases with VWE than we were able to detect in our study setting.

Another limitation might be that we also included patients as GCA-positive who were diagnosed clinically by fulfillment of the American College of Rheumatology criteria but not through TAB. However, TAB is a very invasive technique; therefore, we consider this approach reasonable. Also, there was no significant difference found in patients diagnosed clinically and patients diagnosed through TAB. In addition, sections of the intracavernous ICA were not evaluated for mural inflammation, even though these have been reported more frequently in postmortem cases. ${ }^{5} \mathrm{Nev}$ ertheless, in our opinion, this ICA section is not reliably assessable, given that it is accompanied by venous structures yielding 
hyperintense signal in postcontrast T1WI, indistinguishable from VWE. Additionally, ratings for extra- and intracranial VWE were performed by different raters, and in case of extracranial VWE evaluation, consensus rating was achieved by concomitant reevaluation, while in cases of intracranial VWE ratings, the consensus was established by a third rater. Nevertheless, inter-rater agreement for extracranial VWE was in line with that in previous studies; therefore, we consider our ratings valid. In addition, we did not perform quantitative measurements of the amount of enhancement before and after contrast enhancement. Nevertheless, visual evaluation of contrast enhancement of the extra- or intracranial vessel wall is a standard procedure in clinical routine and is performed as standard in many published studies. Therefore, we consider this approach reasonable. Another limitation might be that in theory, contrast enhancement of arterial vessel walls can also be caused by atherosclerotic plaques. However, these tend to enhance only on 1 side of the vessel wall in comparison with the circular enhancement we observed. Nevertheless, some of the observed changes might also, at least in part, be induced by atherosclerotic plaques.

\section{CONCLUSIONS}

VWE as a sign of mural inflammation of extracranial vessels is a frequent finding in GCA. Additionally, VWE of intradural arteries, mainly the ICA, can be regularly found in patients with GCA. VWE of intradural arteries observed in patients with GCA does not seem to be associated with vessel occlusion or stenosis and cerebral infarction. Mural inflammation of the intradural ICA detected in MR imaging might therefore identify a subgroup of patients with GCA. Therefore, clinical significance (eg, the prognostic value of VWE of the intradural ICA) should be evaluated further in clinical studies.

Disclosures: Caspar Brekenfeld_UNRELATED: Payment for Lectures (including service on Speakers Bureaus): Codman, Comments: preparation and performance of lecture. Jens Fiehler-UNRELATED: Consultancy: Stryker, Codman, MicroVention; Grants/Grants Pending: MicroVention; Payment for Lectures (including service on Speakers Bureaus): Stryker, Codman, MicroVention, Penumbra, Philips Healthcare, Covidien; Travel/Accommodations/Meeting Expenses Unrelated to Activities Listed: Covidien. Thorsten A. Bley-RELATED: Grant: Deutsche Forschungsgesellschaft grant: BL 1132/1-1; UNRELATED: Payment for Lectures (including service on Speakers Bureaus): Bracco, Siemens, Guerbet, Bayer, GE Healthcare.

\section{REFERENCES}

1. Salvarani C, Cantini F, Hunder GG. Polymyalgia rheumatica and giant-cell arteritis. Lancet 2008;372:234-45

2. Weyand CM, Goronzy JJ. Medium- and large-vessel vasculitis. N Engl J Med 2003;349:160-69

3. Maksimowicz-McKinnon K, Clark TM, Hoffman GS. Takayasu arteritis and giant cell arteritis: a spectrum within the same disease? Medicine 2009;88:221-26

4. Blockmans D. Diagnosis and extension of giant cell arteritis: contribution of imaging techniques. Presse Med 2012;41:948-54

5. Wilkinson IM, Russell RW. Arteries of the head and neck in giant cell arteritis: a pathological study to show the pattern of arterial involvement. Arch Neurol 1972;27:378-91

6. Salvarani C, Giannini C, Miller DV, et al. Giant cell arteritis: involvement of intracranial arteries. Arthritis Rheum 2006;55:985-89

7. Bley TA, Uhl M, Carew J, et al. Diagnostic value of high-resolution
MR imaging in giant cell arteritis. AJNR Am J Neuroradiol 2007; 28:1722-27

8. Bley TA, Weiben $\mathrm{O}$, Uhl M, et al. Assessment of the cranial involvement pattern of giant cell arteritis with $3 \mathrm{~T}$ magnetic resonance imaging. Arthritis Rheum 2005;52:2470-77

9. Küker W. Cerebral vasculitis: imaging signs revisited. Neuroradiology 2007;49:471-79

10. Küker W, Gaertner S, Nagele T, et al. Vessel wall contrast enhancement: a diagnostic sign of cerebral vasculitis. Cerebrovasc Dis 2008;26:23-29

11. Bley TA, Geiger J, Jacobsen S, et al. High-resolution MRI for assessment of middle meningeal artery involvement in giant cell arteritis. Ann Rheum Dis 2009;68:1369-70

12. Hunder GG, Bloch DA, Michel BA, et al. The American College of Rheumatology 1990 criteria for the classification of giant cell arteritis. Arthritis Rheum 1990;33:1122-28

13. Landis JR, Koch GG. The measurement of observer agreement for categorical data. Biometrics 1977;33:159-74

14. Bley TA, Uhl M, Venhoff N, et al. 3-T MRI reveals cranial and thoracic inflammatory changes in giant cell arteritis. Clin Rheumatol 2007;26:448-50

15. Bley TA, Wieben $O$, Vaith $P$, et al. Magnetic resonance imaging depicts mural inflammation of the temporal artery in giant cell arteritis. Arthritis Rheum 2004;51:1062-63, author reply 1064

16. Markl M, Uhl M, Wieben O, et al. High resolution 3T MRI for the assessment of cervical and superficial cranial arteries in giant cell arteritis. J Magn Reson Imaging 2006;24:423-27

17. Bley TA, Reinhard M, Hauenstein C, et al. Comparison of duplex sonography and high-resolution magnetic resonance imaging in the diagnosis of giant cell (temporal) arteritis. Arthritis Rheum 2008;58:2574-78

18. Browne L, Hardiman O, O’Dwyer H, et al. Intracranial giant cell arteritis with fatal middle cerebral artery territory infarct. Clin Neuropathol 2003;22:199-203

19. Büttner T, Heye N, Przuntek H. Temporal arteritis with cerebral complications: report of four cases. Eur Neurol 1994;34:162-67

20. Mclean CA, Gonzales MF, Dowling JP. Systemic giant cell arteritis and cerebellar infarction. Stroke 1993;24:899-902

21. Caselli RJ, Hunder GG, Whisnant JP. Neurologic disease in biopsyproven giant cell (temporal) arteritis. Neurology 1988;38:352-59

22. González-Gay MA, Blanco R, Rodriguez-Valverde V, et al. Permanent visual loss and cerebrovascular accidents in giant cell arteritis: predictors and response to treatment. Arthritis Rheum 1998;41: 1497-504

23. Bogousslavsky J, Deruaz JP, Regli F. Bilateral obstruction of internal carotid artery from giant-cell arteritis and massive infarction limited to the vertebrobasilar area. Eur Neurol 1985;24:57-61

24. Butt Z, Cullen JF, Mutlukan E. Pattern of arterial involvement of the head, neck, and eyes in giant cell arteritis: three case reports. $\mathrm{Br} J$ Ophthalmol 1991;75:368-71

25. Cardell BS, Hanley T. A fatal case of giant-cell or temporal arteritis. J Pathol Bacteriol 1951;63:587-97

26. Cull RE. Internal carotid artery occlusion caused by giant cell arteritis. J Neurol Neurosurg Psychiatry 1979;42:1066-67

27. Sheehan MM, Keohane C, Twomey C. Fatal vertebral giant cell arteritis. J Clin Pathol 1993;46:1129-31

28. Thielen KR, Wijdicks EF, Nichols DA. Giant cell (temporal) arteritis: involvement of the vertebral and internal carotid arteries. Mayo Clin Proc 1998;73:444-46

29. Ahdab R, Thabuy F, Menager de Froberville E, et al. Reversible vertebral artery stenosis following corticotherapy in giant cell arteritis. Eur Neurol 2008;59:331-31

30. Kozuka S, Iguchi I, Furuse M, et al. Cerebral aneurysm induced by giant cell arteritis: a case report. Angiology 1979;30:131-37 